\title{
Discharge properties of neurons recorded in the parvalbumin-positive (PV1) nucleus of the rat lateral hypothalamus
}

\author{
Alessandra Lintas ${ }^{\mathrm{a}, \mathrm{b}, *}$ \\ a Department of Medicine/Unit of Anatomy, University of Fribourg, Switzerland \\ ${ }^{\mathrm{b}}$ Neuroheuristic Research Group, HEC Lausanne, University of Lausanne, Switzerland
}

\section{H I G H L I G H T S}

- Random-like firing characterized the majority of PV1 cells.

- Oscillations in the delta range were observed in the posterior part of PV1 nucleus.

- The asynchronous activity produces a network-driven effect on the PV1-target area.

\section{Introduction}

Several types of cells intermingled with the axons of the medial forebrain bundle are located in the lateral hypothalamic area (LHA). These cell groups have tendency to be widely dispersed, rather than confined within anatomically distinct nuclei, despite the fact they often form groups of functionally related neurons [1,2]. On the contrary to this tendency, in the lateral hypothalamus of rodents a well identified cluster of parvalbumin (PV)-positive neurons has been described [3]. In rodents the PV-positive neurons represent the vast majority of the cells belonging to a clearly distinct cytoarchitectonically and neurochemically defined lateral hypothalamic area, for

\footnotetext{
* Correspondence to: University of Lausanne, Internef 138.2, CH-1015 Lausanne, Switzerland. Tel.: +41216923587.

E-mail addresses: Alessandra.Lintas@neuroheuristic.org, Alessandra.Lintas@unil.ch
}

they are considered to form an entity termed PV1-nucleus [4,5]. In primates this region is referred to as the lateral tuberal nucleus (LTN) [7].

Three cell types were observed in the rodent PV1-nucleus: small PV immunoreactive neurons preferentially located in the anterior part of the nucleus, large PV immunoreactive neurons preferentially located in the posterior part of the nucleus, and PV-negative neurons [4]. According to topographical mapping of the gene expression and double-labeling for glutamate and for PV it is extremely likely that the PV-positive neurons of the PV1nucleus are glutamatergic projecting neurons $[4,8]$. Glutamate is an excitatory amino acid assumed to represent the main neurotransmitter used for distribution and transmission of information in the brain [9]. The recent study of the PV1-nucleus efferent projections revealed that its major target is a narrow column of terminal fields located ipsilaterally at the edge of the periaqueductal gray (PAG), ventrolateral to the aqueduct, not coinciding with any known subdivision of the PAG [5]. It is interesting to notice that 
elsewhere in the brain PV-stained neurons correspond to GABAergic cells [3] mediating an inhibitory effect through GABA(A) receptors, thus exerting a regulatory function, either via a direct inhibition or an indirect disinhibition [10]. Hence, GABAergic neurons and PV are generally associated and their role in controlling synaptic plasticity, modulating the firing pattern, and the spiketiming-dependent plasticity has been clearly established [11-15]. The fact that the PV1-nucleus is likely to be composed by glutamatergic cells expressing PV and that its main target is a well delimited column of cells on the edge of the ipsilateral PAG raises the question whether its function is to integrate, relay and transmit an information or rather to regulate the activity of the target cells. The aim of this study is to investigate PV1-nucleus firing pattern and to suggest its functional role.

\section{Materials and methods}

All experimental procedures were conducted in accordance with ethical principles and guidelines for experiments on animals mandated by the Swiss Academy of Medical Sciences and Swiss Academy of Sciences (3rd ed., 2005) under control of the Veterinary Commission for Animal Research of the Canton of Fribourg, Switzerland.

\subsection{Subjects and surgical procedure}

Adult Wistar rats were housed in pairs on a $12 \mathrm{~h} / 12 \mathrm{~h}$ light/dark cycle, and food and water available ad libitum. The rats, weighing $280-350 \mathrm{~g}$, were anesthetized with a mixture of ketamine $(75 \mathrm{mg} / \mathrm{kg} \mathrm{BW})$ and xylazine $(10 \mathrm{mg} / \mathrm{kg} \mathrm{BW})$ diluted in saline. All surgical wounds were infiltrated subcutaneous with Scandicaine $0.5 \%$ (AstraZeneca) for local anesthesia. The animals were mounted in a stereotaxic frame, a hole was drilled in the skull and one microelectrode was advanced vertically by $5 \mu \mathrm{m}$ steps aimed to the lateral hypothalamic PV1-nucleus [4]. The body temperature was monitored and maintained in the range $38-39^{\circ} \mathrm{C}$. The pedal withdrawal reflex was periodically checked and supplemental doses of ketamine were provided during the whole recording session if necessary.

\subsection{Electrophysiological recordings}

The recordings were performed in the left hemisphere with glass-coated platinum-plated tungsten microelectrodes having an impedance in the range $0.5-2 \mathrm{M} \Omega$ measured at a frequency of $1 \mathrm{kHz}$. Signals from the microelectrodes were amplified, filtered $(400 \mathrm{~Hz}-20 \mathrm{kHz})$ viewed on an oscilloscope, and digitally recorded in WAV format $(44,100 \mathrm{~Hz}$ sampling rate, 16 bit resolution) for computerized offline analysis with template matching spike sorting algorithm at a time resolution of $1 \mathrm{~ms}$ [16]. The first recording session started approximately $90 \mathrm{~min}$ after the end of the surgical preparation. The data were gathered during spontaneous activity, i.e. in the absence of any operator-induced stimulation, for a continuous interval of 300-600 s. All recordings started at least $15 \mathrm{~min}$ after any supplementary dose of anesthetic and terminated at least 20 min before a new injection, thus assuming the recording conditions corresponded to a steady level of anesthesia.

\subsection{Histological and immunohistological procedures}

At the end of the recording sessions (lasting 4-6h) electrolytic lesions were placed at specific depths of the electrode track using 10 current pulses of $8 \mu \mathrm{A}$ for $7 \mathrm{~s}$ at regular intervals of $10 \mathrm{~s}$. At the conclusion of the experiments, animals were deeply anesthetized and transcardially perfused with isotonic saline immediately followed by fixative solution ( $4 \%$ paraformaldehyde in phosphate buffer

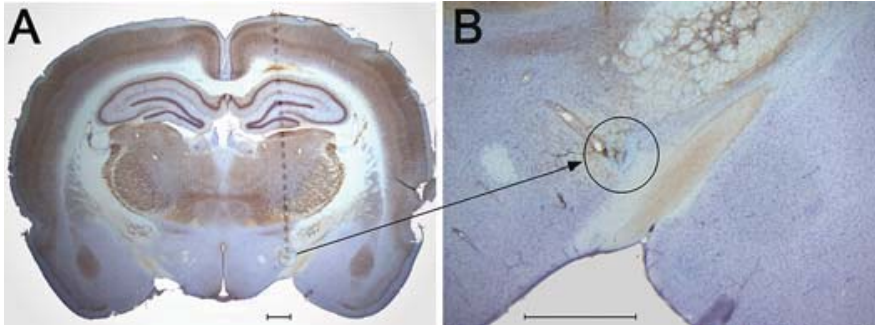

Fig. 1. Histological analysis. (A) Microphotograph of a coronal section (at Interaural level 6.1 stained with cresyl violet and PV-immunostaining showing a representative electrode penetration aiming the PV1-nucleus. The entire electrode track is represented by a dotted line. (B) Enlargement of the previous panel emphasizing the electrolytic lesion within the PV1-nucleus at the center of the circle. Scale bar is $1 \mathrm{~mm}$.

$0.1 \mathrm{M}, \mathrm{pH} 7.3)$. Brains were removed and placed in $18 \%$ solution of sucrose in phosphate buffered containing $0.1 \%$ sodium azide for one day at $4{ }^{\circ} \mathrm{C}$. They were then frozen in pulverized dry ice. The specimens were cryosectioned into $50 \mu \mathrm{m}$-thick sections and collected in $0.1 \mathrm{M}$ phosphate buffer ( $\mathrm{pH}$ 7.3). Immunofluorescence- and immunoperoxidase-staining techniques were conducted according to published protocols [4]. The sections were stained with cresyl violet for the reconstruction of the electrode tracks and localization of the electrolytic lesions.

\subsection{Statistical analysis}

Spike trains were analyzed by renewal density histograms scaled in rate units (spikes/s). For each histogram, the 99\% confidence limits were calculated, assuming that spike occurrences followed a Poisson distribution. The Fano factor (equal to 1 for data following a Poisson process) was used to characterize the variability of the spike train [17]. Statistical analyses were performed with the R Project for Statistical Computing (http://www.r-project.org/).

\section{Results}

Animals found to have tracks with placements outside of the targeted area corresponding to the PV1-nucleus were excluded from analysis. The final sample included a total of 15 electrode penetrations performed in the left hemisphere of 8 rats. Taking into consideration the tissue retraction during the histological processing, the backlash of the electrode advancement and the stereotaxic positioning of the electrodes, it is assumed that the site of a single unit recording can be estimated with a margin of $50-80 \mu \mathrm{m}$ of uncertainty along the vertical track. Fig. 1 illustrates an example of a recording track with an electrolytic lesion within the PV1-nucleus. A total of 30 single units were clearly localized in the area of the PV1-nucleus. These cells were characterized by stable firing activity, i.e. same firing rate during the first $100 \mathrm{~s}$ and the last $100 \mathrm{~s}$ of the recording session. Additional 25 single units were recorded along the same tracks, but their location was clearly not in the PV1-nucleus after histological check.

Three types of firing patterns of PV1 cells were identified during the spontaneous activity by the analysis of the autocorrelograms. The first pattern is typical of those neurons with a constant probability to spike, corresponding to a flat autocorrelogram, thus forming the "regular" (REG) type class of cells (Fig. 2A). This class was the most frequently observed $(70 \%, n=21)$ with a median firing rate equal to $1.7 \mathrm{spikes} / \mathrm{s}$. Cells showing a tendency to deviate from a constant probability of firing were classified either in "bursting cells" (BC, $n=5$ ), characterized by a hump in the autocorrelogram near time zero (Fig. 2C), or in "oscillatory cells" (OSC, $n=4$ ), in the range $1.6-2.1 \mathrm{~Hz}$ (Fig. 2D). 
A
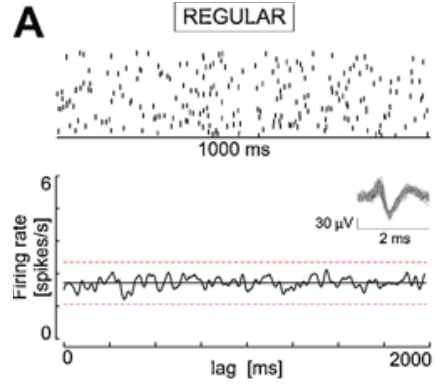

B

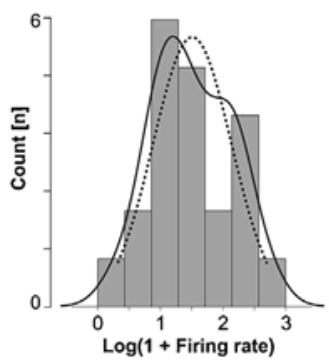

C

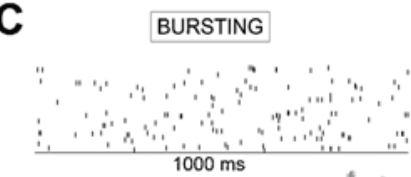

D

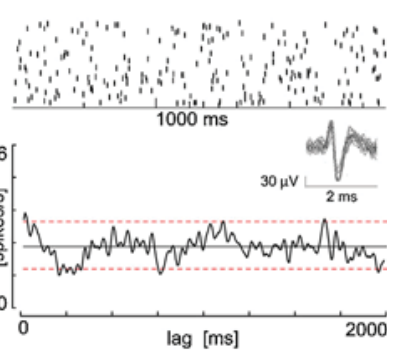

E

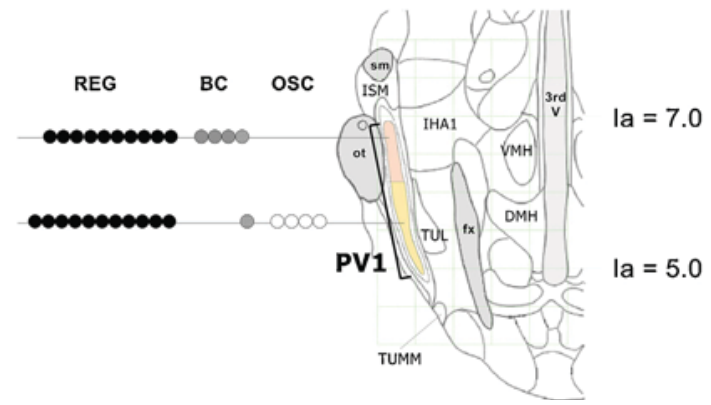

Fig. 2. Firing patterns during spontaneous activity. (A, C, D) In the upper part of each panel there is a raster display of the spike train and in the lower part there are the oscilloscope trace of the extracellularly recorded single unit and the autocorrelogram smoothed by a Gaussian bin of $25 \mathrm{~ms}$ showing the firing rate (spikes/s) as a function of the lag (ms). (A) A regular cell (\#PA1D02c1). (B) Distribution (in logarithmic scale) of the regular cells' firing rate with the kernel density plot (solid line) and the Gaussian fit (dotted line). (C) A bursting cell (\#PA1D01nc3). (D) An oscillatory cell (\#PA6D03c2). (E) Distribution of PV1 cells types (REG, black dots; BC, grey dots; OSC, white dots) along the rostro-caudal axis, (Interaural coordinates 5.0-7.0). Abbreviations: 3dv: third ventricule; DMH: dorsomedial hypothalamic nucleus; fx: fornix; IHA1: part of the intermediate hypothalamic area; ISM: interstitial nucleus of the stria terminalis; ot: optic tract; sm: medullary stria; TUL: lateral tuberal nucleus; TUMM: tuberomammillary nucleus; VMH: ventromedial hypothalamic nucleus. Modified from [4].

Table 1 shows the values of the firing rates and Fano factor for all cell classes. The firing rates were not Gaussian distributed (D'Agostino-Pearson normality test, omnibus $K^{2}=2.97, p<0.01$ ), but taking the logarithm of the firing rates of all cell groups we observed a normal distribution (D'Agostino-Pearson normality test, omnibus $K^{2}=1.61, p=0.45$ ). It is interesting to notice the

Table 1

Firing rate (median, mean \pm S.E.M.) and Fano factor of PV1-nucleus spike trains. Statistics are described in the text.

\begin{tabular}{lllll}
\hline Cell type & Total & REG & BC & OSC \\
\hline$N$ & 30 & 21 & 5 & 4 \\
& $(100 \%)$ & $(70 \%)$ & $(17 \%)$ & $(13 \%)$ \\
Firing rate & 1.7 & 1.7 & 1.5 & 2.5 \\
(spikes/s) & $(2.2 \pm 0.2)$ & $(2.0 \pm 0.2)$ & $(2.2 \pm 0.9)$ & $(2.9 \pm 0.8)$ \\
Fano factor & 1.0 & 1.0 & 1.6 & 1.1 \\
& $(1.1 \pm 0.1)$ & $(1.0 \pm 0.1)$ & $(1.6 \pm 0.1)$ & $(1.1 \pm 0.1)$
\end{tabular}

log-normal distribution of the firing rates of class REG neurons (Fig. 2B). A Fano factor value near 1 suggests that the dynamics of the processes producing the neuronal discharge is essentially random. The REG and OSC Fano factor values were Gaussian distributed (D'Agostino-Pearson normality test, omnibus $K^{2}=1.26$, $p=0.53)$.

The firing rates of the three cell groups were compared with a one-way ANOVA. Bartlett's test did not show a violation of homogeneity of variances $\left(K^{2}(2)=1.12, p=0.57\right)$ and no significant effect was found of the cell type on the logarithmic distribution of the firing rate $((2,27)=0.70, p=0.51)$. The comparison between the three groups using nonparametric Kruskal-Wallis test revealed a significant effect of the cell type on Fano factor $\left(\chi^{2}=12.34, p<0.01\right)$. A post-hoc test using Mann-Whitney tests with Holm-Bonferroni correction showed no difference between REG and OSC cells and significant differences between OSC and BC cells $(p<0.05, r=0.66)$ and between REG and BC cells $(p<0.01, r=0.82)$. These results should nevertheless be considered with caution because the sample size of BC and OSC groups is extremely small. The PV1 nucleus was subdived on the basis of the anatomical sampling. The four most anterior tracks (in the Interaural range 6.3-6.8) and the four most posterior tracks (in the Interaural range 5.4-6.2) were grouped in two samples. Fig. 2E shows that regular cells were evenly sampled throughout the nucleus, whereas the $\mathrm{BC}$ cells tended to appear in the anterior part and OSC cells in the posterior part. The likelihood of obtaining the observed distributions of firing patterns across the two parts of PV1-nucleus was estimated by computing the likelihood-ratio statistics $\left(\chi^{2}=7.39, p<0.05\right)$ that rejected the hypothesis that no difference existed between the anterior and posterior part.

Pairs of cells were recorded from the same electrode tip in 8 sites. In two of these sites three cells were recorded simultaneously. Overall thirteen crosscorrelograms could be computed and all were characterized by a flat curve, thus showing the independance of firing and no interaction between pairs of simultaneously recorded cells.

\section{Discussion}

The main finding of this study is that regular cells, with a median firing rate of 1.7 spikes/s and Fano factor equal to 1, represent the typical class (70\% of the recordings) of PV1 cells evenly distributed along the rostro-caudal axis. The regular cells firing pattern is characterized by a random spike train so that successive spike intervals are statistically independent and generate a flat autocorrelogram [18]. Pairs of cells recorded simultaneously did not show any sign of functional interaction and four cells exhibiting an oscillatory activity within a narrow range of the $\delta$-frequency band $(0.5-4 \mathrm{~Hz})$ were observed only in the posterior part of the PV1-nucleus.

If a neuron fires randomly, it is likely to have little effect, if any, on its target unless its activity is time-locked to a series of other spikes converging to the same cell [19]. Asynchronous activity in an unstructured, sparsely connected network with weak synaptic couplings falls in a state such that an external input may favor information transmission in the target structure $[20,21]$ and trigger the transition of an activity pattern in a neural network [22-24]. The random firing properties of PV1 neurons suggest that glutamate is released asynchronously at synaptic terminals and that "network driven" PV1 excitatory activity might contribute to achieve temporal frequency modulation of selected patterns of activity [25-27]. Notice that all cells were recorded under general anesthesia induced by ketamine and xylazine and not by urethane, but this does not discard that quite different activity could occur during normal behavior. 
Spontaneous activity is determined by a combination of circuit and cellular electrophysiological properties. Sources of cellular noise include the ion channels of excitable membranes, synaptic transmission and network interactions. Despite the dominance of $\mathrm{K}^{+}$currents the contribution of Ca-activated $\mathrm{K}$ currents to the resting potential of neurons is widely recognized, particularly in PV-expressing neurons firing randomly and in burst $[14,28]$ Large-conductance calcium-activated potassium channels (BK), small-conductance $\mathrm{Ca}^{2+}$-activated potassium channels (SK) and $\mathrm{Ca}^{2+}$-activated nonselective cation channels regulate spontaneous firing in a complementary manner $[29,30]$. Parvalbumin is a $\mathrm{Ca}^{2+}$ buffer protein characterized by a slow-onset $\mathrm{Ca}^{2+}$ binding that generally does not affect the initial amplitude of $\mathrm{Ca}^{2+}$ transients, but then accelerates the early phase of the intracellular $\mathrm{Ca}^{2+}$ concentration, thus affecting short-term plasticity and controlling the intracellular $\mathrm{Ca}^{2+}$ available to SK channels [30]. Loss of PV leads to enhanced susceptibility to epileptic seizure [12] and modifications of the firing patterns at the thalamocortical level $[13,15]$. The question is raised whether firing patterns other than the regular firing correspond to distinct cell populations, maybe lying in the border of the nucleus, or whether they are generated by cells that are in a different functional state.

Three cell types, namely small and large PV-positive neurons as well as PV-negative ones, were reported in PV1-nucleus [6,4]. Despite the small numbers of our samples the few cells $(n=4)$ characterized by activity in the $\delta$-frequency range were all recorded in the posterior part of the nucleus. The experimental method used here does not allow to identify which cell type is associated to which firing pattern, but the largest observed amplitudes of the extracellularly recorded spike waveforms were those of the oscillatory cells. The spike amplitude is roughly proportional to the sum of the cross-sectional areas of the dendrites connected to the cell body [31]. Thus, larger neurons are likely to generate extracellular spikes with larger amplitudes and the large PV immunoreactive neurons were preferentially reported in the posterior part of the nucleus [4].

The few oscillatory cells were observed with frequencies associated with deep sleep. Interestingly, in the dendrites of other PV-positive cells (located in the thalamic reticular nucleus) the interplay of SK channels, transient voltage-gated calcium channels ( T channels), and sarcoendoplasmic reticulum calcium transport ATPases comprises a specialized $\mathrm{Ca}^{2+}$ signaling triad to regulate oscillatory dynamics related to sleep [32]. The current continuous recording time for one cell was at most $20 \mathrm{~min}$, well below the ultradian rhythm of 1 cycle per $100 \mathrm{~min}$ [33]. The possibility that the oscillatory cells are not part of a separate population, but cells in a particular state of sleep, cannot be discarded a priori, in spite of the fact that no cell was observed switching between regular and oscillatory firing modes.

In Huntington's disease [34] and Pick's disease [35] selective neuropathological changes were observed in the human LTN. Despite different etiology both diseases exhibit progressive impairement of speech production akin of speech apraxia accompanied by a decrease in cognitive abilities leading to dementia akin of frontotemporal dementia. No precise functions are known to be associated with the area of the PAG reached by PV1-nucleus efferent projections. However, it is worth reporting that lesions of PAG affect states of consciousness [36] and that PAG receives afferences from the prefrontal cortex [37] and projects to intralaminar and midline thalamic nuclei [38]. Moreover, PV is highly expressed in the thalamic reticular nucleus [13] which plays a key role in 'gating' consciousness [39]. These observations taken together with the present findings may suggest that the activity of PV1-nucleus regulates psychomotor functions in the framework of an extended reticular thalamic activating system.

\section{Conclusions}

It is possible to speculate that the asynchronous activity characteristic of the PV1 neurons is generated intrinsically, subject to modulation by synaptic inputs, intracellular $\mathrm{Ca}^{2+}$ concentrations, and that the "network-driven" effect on their target may produce various firing patterns with transitions induced through small changes in PV1 neuronal activity. The hypothesis is raised that the firing pattern of PV1-nucleus participates to functional network activity controlling dynamic information related to condition transitions associated with awareness and non-conscious perception.

\section{Acknowledgments}

The author thanks M.R. Celio, A.E.P. Villa, B. Schwaller and R. Kretz for their suggestions and M. Kaczorowski, C. Marti and S. Eichenberger for their technical assistance.

\section{References}

[1] J.C. Sipe, R.Y. Moore, The lateral hypothalamic area, Cell Tissue Res. 179 (1977) 177-196.

[2] C. Saper, L. Swanson, W. Cowan, An autoradiographic study of the efferent connections of the lateral hypothalamic area in the rat, J. Comp. Neurol. 183 (1979) 689-706

[3] M.R. Celio, Calbindin D-28k and Parvalbumin in the rat nervous system, Neuroscience 35 (1990) 375-475.

[4] Z. Mészár, F. Girard, C.B. Saper, M.R. Celio, The lateral hypothalamic parvalbumin-immunoreactive (PV1) nucleus in rodents, J. Comp. Neurol. 520 (2012) 798-815

[5] M.R. Celio, A. Babalian, Q.H. Ha, S. Eichenberger, L. Clément, C. Marti, C.B. Saper Efferent connections of the parvalbumin-positive (PV1) nucleus in the lateral hypothalamus of rodents, J. Comp. Neurol. 521 (2013) 3133-3153.

[6] L.M. Geeraedts, R. Nieuwenhuys, J.G. Veening, Medial forebrain bundle of the rat: IV. Cytoarchitecture of the caudal (lateral hypothalamic) part of the medial forebrain bundle bed nucleus, J. Comp. Neurol. 294 (1990) 537-568.

[7] R. Bleier, P. Cohn, I.R. Siggelkow, A cytoarchitectonic atlas of the hypothalamus and hypothalamic third ventricle of the rat, in: P.J. Morgane, J. Panksepp (Eds.) Handbook of the Hypothalamus, Anatomy of the Hypothalamus, vol. 1, Marcel Dekker, Inc, New York, 1979, pp. 137-220.

[8] F. Girard, Z. Meszar, C. Marti, F.P. Davis, M. Celio, Gene expression analysis in the parvalbumin-immunoreactive PV1 nucleus of the mouse lateral hypothalamus, Eur. J. Neurosci. 34 (2011) 1934-1943.

[9] C.G. Parsons, W. Danysz, W.Zieglgänsberger, Excitatory amino acid neurotransmission, Handb. Exp. Pharmacol. 169 (2005) 249-303.

[10] J.M. Tepper, C.J. Wilson, T. Koós, Feedforward and feedback inhibition in neostriatal gabaergic spiny neurons, Brain Res. Rev. 58 (2008) 272-281.

11] O. Caillard, H. Moreno, B. Schwaller, I. Llano, M.R. Celio, A. Marty, Role of the calcium-binding protein parvalbumin in short-term synaptic plasticity, Proc. Natl. Acad. Sci. U.S.A. 97 (2000) 13372-13377

[12] B. Schwaller, I.V. Tetko, P. Tandon, D.C. Silveira, M. Vreugdenhil, T. Henzi, M.C. Potier, M.R. Celio, A.E. Villa, Parvalbumin deficiency affects network properties resulting in increased susceptibility to epileptic seizures, Mol. Cell. Neurosci. 25 (2004) 650-663.

[13] L. Albéri, A. Lintas, R. Kretz, B. Schwaller, A.E. Villa, The calcium-binding protein parvalbumin modulates the firing properties of the reticular thalamic nucleus bursting neurons, J. Neurophysiol. 109 (2013) 2827-2841.

[14] D. Orduz, D.P. Bischop, B. Schwaller, S.N. Schiffmann, D. Gall, Parvalbumin tunes spike-timing and efferent short-term plasticity in striatal fast spiking interneurons, J. Physiol. 591 (2013) 3215-3232.

[15] A. Lintas, B. Schwaller, A.E.P. Villa, Visual thalamocortical circuits in parvalbumin-deficient mice, Brain Res. 1536 (2013) 107-118.

[16] Y. Asai, T. Aksenova, A.E.P. Villa, On-line real-time oriented application for neuronal spike sorting with unsupervised learning, Lect. Notes Comput. Sci. 3696 (2005) 109-114

[17] L. Sacerdote, A.E. Villa, C. Zucca, On the classification of experimental data modeled via a stochastic leaky integrate and fire model through boundary values, Bull. Math. Biol. 68 (2006) 1257-1274.

[18] G.P. Moore, D.H. Perkel, J.P. Segundo, Statistical analysis and functional interpretation of neuronal spike data, Annu. Rev. Physiol. 28 (1966) 493-522.

[19] Y. Yarom, J. Hounsgaard, Voltage fluctuations in neurons: signal or noise? Physiol. Rev. 91 (2011) 917-929.

[20] A.A. Faisal, L.P. Selen, D.M. Wolpert, Noise in the nervous system, Nat. Rev. Neurosci. 9 (2008) 292-303.

[21] S. Ostojic, Two types of asynchronous activity in networks of excitatory and inhibitory spiking neurons, Nat. Neurosci. 17 (2014) 594-600.

[22] R.R. Llinás, The intrinsic electrophysiological properties of mammalian neurons: insights into central nervous system function, Science 242 (1988) 1654-1664. 
[23] D.J. Amit, A. Treves, Associative memory neural network with low temporal spiking rates, Proc. Natl. Acad. Sci. U.S.A. 86 (1989) 78717875.

[24] M. Häusser, I.M. Raman, T. Otis, S.L. Smith, A. Nelson, S. du Lac, Y. Loewenstein, S. Mahon, C. Pennartz, I. Cohen, Y. Yarom, The beat goes on: spontaneous firing in mammalian neuronal microcircuits, J. Neurosci. 24 (2004) 92159219.

[25] J. Iglesias, A.E.P. Villa, Effect of stimulus-driven pruning on the detection of spatiotemporal patterns of activity in large neural networks, BioSystems 89 (2007) 287-293.

[26] J.P. Segundo, What can neurons do to serve as integrating devices? J. Theor. Neurobiol. 5 (1986) 1-59.

[27] Y. Chen, H. Zhang, H. Wang, L. Yu, Y. Chen, The role of coincidence-detector neurons in the reliability and precision of subthreshold signal detection in noise, PLoS ONE 8 (2013) e56822.

[28] P. Coulon, D. Herr, T. Kanyshkova, P. Meuth, T. Budde, H.C. Pape, Burst discharges in neurons of the thalamic reticular nucleus are shaped by calcium-induced calcium release, Cell Calcium 46 (2009) 333-346.

[29] J.A. Goldberg, C.J. Wilson, Control of spontaneous firing patterns by the selective coupling of calcium currents to calcium-activated potassium currents in striatal cholinergic interneurons, J. Neurosci. 25 (2005) 10230-10238.

[30] B. Schwaller, The continuing disappearance of "pure" $\mathrm{Ca}^{2+}$ buffers, Cell. Mol. Life Sci. 66 (2009) 275-300.

[31] K.H. Pettersen, G.T. Einevoll, Amplitude variability and extracellular low-pass filtering of neuronal spikes, Biophys. J. 94 (2008) 784-802.
[32] L. Cueni, M. Canepari, R. Lujan, Y. Emmenegger, M. Watanabe, C.T. Bond, P. Franken, J.P. Adelman, A. Luthi, T-type $\mathrm{Ca}^{2+}$ channels, SK2 channels and SERCAs gate sleep-related oscillations in thalamic dendrites, Nat. Neurosci. 11 (2008) 683-692.

[33] K. Grass, H. Prast, A. Philippu, Ultradian rhythm in the delta and theta frequency bands of the EEG in the posterior hypothalamus of the rat, Neurosci. Lett. 191 (1995) 161-164.

[34] H.P. Kremer, R.A. Roos, G.M. Dingjan, G.T. Bots, G.W. Bruyn, M.A. Hofman, The hypothalamic lateral tuberal nucleus and the characteristics of neuronal loss in Huntington's disease, Neurosci. Lett. 132 (1991) 101-104.

[35] H. Braak, E. Braak, Pick's disease: cytoskeletal changes in the hypothalamic lateral tuberal nucleus, Brain Res. 802 (1998) 119-124.

[36] E.E. Benarroch, Periaqueductal gray: an interface for behavioral control, Neurology 78 (2012) 210-217.

[37] S.R. Sesack, A.Y. Deutch, R.H. Roth, B.S. Bunney, Topographical organization of the efferent projections of the medial prefrontal cortex in the rat: an anterograde tract-tracing study with Phaseolus vulgaris leucoagglutinin, J. Comp. Neurol. 290 (1989) 213-242.

[38] A.A. Cameron, I.A. Khan, K.N. Westlund, K.D. Cliffer, W.D. Willis, The efferent projections of the periaqueductal gray in the rat: a Phaseolus vulgarisleucoagglutinin study. I. Ascending projections, J. Comp. Neurol. 351 (1995) 568-584.

[39] F. Crick, Function of the thalamic reticular complex: the searchlight hypothesis, Proc. Natl. Acad. Sci. U.S.A. 81 (1984) 4586-4590. 